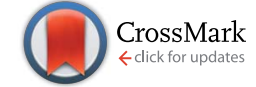

Cite this: RSC Adv., 2017, 7, 2842

Received 12th November 2016 Accepted 18th December 2016

DOI: $10.1039 / c 6 r a 26684 c$

www.rsc.org/advances

\section{Preparation of cellulose-based fluorescent carbon nanoparticles and their application in trace detection of $\mathrm{Pb}(\mathrm{II})$}

\author{
Zhang Yun-fei, Halidan Maimaiti* and Zhang Bo
}

Nanocrystalline cellulose (NCC) with a particle size of $23.80 \pm 0.33 \mathrm{~nm}$ was prepared from microcrystalline cellulose by a mixed treatment with acids and ultrasound. The NCC was chlorinated with thionyl chloride, submitted to dehydrating carbonization and oxidization, and then modified with ethylenediamine (EDA) to obtain fluorescent carbon nanoparticles (NCC-EDA). These were characterized by elemental analysis, TEM, BET, FT-IR, XRD, TG-DTA, XPS, UV-Vis, and photoluminescence. It was found that NCC-EDA were vesiclelike porous particles formed through the linking and intertwining effects of EDA on the $\mathrm{SOCl}_{2}$-treated NCC. The surface of NCC-EDA is rich in oxygen-, nitrogen- and sulfur-containing functional groups, and the particle size was $41.45 \pm 0.55 \mathrm{~nm}$. This endows the NCC-EDA with good dispersity in water, high absorbance and strong fluorescence. The NCC-EDA are shown to be viable probes in that their fluorescence is selectively quenching by $\mathrm{Pb}(॥)$. The findings were exploited to design a fluorometric assay for $\mathrm{Pb}($ ॥) that has a $24 \mathrm{nM}$ detection limit.

\section{Introduction}

In light of the good optical property and water dispersity of fluorescent carbon nanoparticles (CNPs), especially their low toxicity and desirable biocompatibility relative to quantum dots (QDs), ${ }^{1}$ the preparation of fluorescent carbon nanomaterials for special applications by using different raw materials and different methods has drawn extensive attention all over the world..$^{2-4}$ Particularly, the preparation of fluorescent CNPs using environmentally-benign carbohydrates as carbon sources has become the focus of attention in recent years due to the advantages of operation simplicity, short reaction time and high product quantum efficiency. For instance, Sun et al. prepared fluorescent CNPs with relatively high quantum efficiency by treating shaddock peel with a hydrothermal method; ${ }^{5}$ Chang et al. reported a green approach to the one-pot synthesis of fluorescent CNPs with $\mathrm{sp}^{2}$-bonded carbon atoms from coffee grounds; ${ }^{6}$ Han et al. synthesized fluorescent CNPs with high quality through low-temperature carbonization and simple filtration using watermelon peel; Krysmann et al. obtained fluorescent CNPs via high-temperature pyrolysis by using biomass as starting material; ${ }^{8}$ Sarka et al. treated roasted peanut shells at $700{ }^{\circ} \mathrm{C}$ under nitrogen atmosphere for carbonization, and then carried out oxidization experiment in aqueous solution to obtain cellulose-based fluorescent CNPs; ${ }^{9}$ Pereira et al. prepared watersoluble bio-based fluorescent CNPs from cellulose nanocrystals using different pyrolysis temperatures; ${ }^{10}$ Peng et al. treated carbohydrates with concentrated sulfuric acid for dehydrated

Institute of Chemistry and Chemical Industry, Xinjiang University, Urumqi 830046, Xinjiang, China. E-mail:m15899160730@163.com carbonization, and then executed a series of processes including oxidization with nitric acid and deactivation of amine-terminated compounds to obtain fluorescent CNPs; ${ }^{11}$ Yang et al. used chitosan as starting material and prepared amino group functionalized fluorescent CNPs. ${ }^{12}$ In addition, the preparations of fluorescent CNPs using low-molecular-weight carbohydrates such as glucose, sucrose and citric acid have also been reported. ${ }^{13-15}$

It can be seen from the above results that when lowmolecular-weight carbohydrates are used as carbon sources, dehydrated carbonization and subsequent modification are considered as the major approaches to prepare fluorescent CNPs; when high-molecular-weight carbohydrates are used, hydrothermal treatment and high-temperature pyrolysis are usually accepted as the proper methods. Then, is it possible to prepare fluorescent CNPs with identical properties by simple acid treatment on high-molecular-weight carbohydrates and subsequent surface modification, but not rely on high temperature pyrolysis? For this purpose, water-soluble cellulose-based fluorescent carbon nanoparticles (NCC-EDA) with relatively high fluorescence quantum yield were prepared by using cellulose, the most abundant organic raw material in nature, as starting material. On this basis, the structure and fluorescence property of the NCC-EDA were studied, and the NCC-EDA were used as fluorescent probe to detect metal ions in water.

\section{Experiment}

\subsection{Materials and methods}

Microcrystalline cellulose (MCC, with particle size range of 10$100 \mu \mathrm{m}$ and moisture content of $6 \%$ ) of technical grade was 
purchased from Tianjin Guangfu Fine Chemical Co., Ltd. Thionyl chloride $\left(\mathrm{SOCl}_{2}\right)$ of analytical grade was purchased from Aladdin Reagent Co., Ltd. Ethylenediamine (EDA) of analytical grade was purchased from Tianjin Zhiyuan Chemical Reagent Co., Ltd. N,N-Dimethylformamide (DMF), tetrahydrofuran (THF) and absolute ethanol $\left(\mathrm{C}_{2} \mathrm{H}_{5} \mathrm{OH}\right)$ are all of analytical grade and commercially available.

Ready-to-use dialysis bags of Spectrum labs (no. 1: molecular weight cut-off of 100-500; no. 2: molecular weight cut-off of 500-1000) were purchased from Shanghai Toscience Biotechnology Co., Ltd.

\subsection{Preparation of product}

The preparation process of the NCC-EDA is shown in Scheme 1.

2.2.1 Preparation of NCC. $5 \mathrm{~g}$ MCC was placed into a 200 $\mathrm{mL}$ wide-mouth bottle, and then $72 \mathrm{~mL}$ deionized water, $36 \mathrm{~mL}$ concentrated sulfuric acid and $12 \mathrm{~mL}$ concentrated hydrochloric acid were added. After stirring at room temperature for $12 \mathrm{~h}$, the mixture was further dispersed in ultrasound at $60^{\circ} \mathrm{C}$ for another $12 \mathrm{~h}$ and then transferred into no. 1 dialysis bag for dialysis. The dialysis proceeded in water for $72 \mathrm{~h}$ until the $\mathrm{pH}$ was neutral, during which water was constantly refreshed. The solid precipitate was separated from the mixture by high speed centrifugation, and then air drying was conducted to obtain the NCC.

2.2.2 Preparation of NCC-EDA. $1 \mathrm{~g}$ NCC and $20 \mathrm{~mL}$ DMF were placed in a reaction flask and sonicated at room temperature for $2 \mathrm{~h}$ to fully disperse the NCC into an emulsion. Then $\mathrm{SOCl}_{2}$ was added into the emulsion and the mixture was stirred at room temperature for $12 \mathrm{~h}$. Afterwards, the mixture was centrifuged to remove DMF and unreacted $\mathrm{SOCl}_{2}$, and washed twice with THF to generate chlorinated intermediate $\mathrm{NCC}-\mathrm{Cl}$. The $\mathrm{NCC}-\mathrm{Cl}$ was then mixed with EDA and refluxed for $72 \mathrm{~h}$. After reflux reaction, the mixture was cooled down to $60^{\circ} \mathrm{C}$, and distilled water was added into the mixture. Then, the mixture was stirred for $3-4 \mathrm{~h}$ and centrifuged at a speed of $10000 \mathrm{rpm}$ for $10 \mathrm{~min}$. The supernatant was transferred into no. 2 dialysis bags and dialyzed in water for $72 \mathrm{~h}$ to obtain an aqueous dispersion of NCC-EDA. Finally, NCCEDA particles were obtained by vacuum distillation.

\subsection{Characterization of product}

The aqueous dispersions of NCC and NCC-EDA were respectively dropped onto a copper grid and then dried for observing the morphology and structure of the particles by transmission electron microscope (TEM, Hitachi H-600, Japan). The particle sizes of the NCC and NCC-EDA aqueous dispersions were measured by NANO-S90 laser particle size analyzer. The contents of various elements, such as $\mathrm{C}, \mathrm{H}, \mathrm{O}, \mathrm{N}$ and $\mathrm{S}$, in NCC, NCC-Cl and NCC-EDA were determined by FLASHEA-PE2400 elemental analyzer. The specific surface area, pore size and total pore volume of NCC and NCC-EDA were determined by Autosorb-IQ2 surface area and pore size analyzer (Quantachrome, America). FT-IR characterization of starting material and product was performed by Bruker-EQUINOX 55 Fourier transform infrared spectrometer. XRD characterization of NCC, $\mathrm{NCC}-\mathrm{Cl}$, and NCCEDA was carried out by MAC-M18XHF22-SRA X-ray diffractometer. TG-DTA analysis of the starting material and product was performed by PE-DTA/1700 thermal analyzer. The surface state of NCC-EDA was analyzed by ESCALAB 250 X-ray photoelectron spectrometer (XPS Thermo Fisher, America).

\subsection{Optical property characterization of product}

The aqueous dispersion of NCC-EDA was transferred into cuvette after dilution and then measured by UV $3900 \mathrm{H}$ UV-Vis spectrophotometer (Hitachi, Japan) for UV-Vis absorption spectra and by Fluorolog-3 fluorescence spectrometer (Horiba, France) for emission spectra at different excitation wavelengths, respectively.

\subsection{Fluorescence detection of $\mathbf{P b}^{2+}$ by NCC-EDA}

$1 \mathrm{~mL}$ NCC-EDA aqueous dispersion with a concentration of $0.25 \mathrm{~g} \mathrm{~L}^{-1}$ was placed in a $100 \mathrm{~mL}$ volumetric flask, followed by the addition of phosphate buffer to the scale marking. After the solution in the volumetric flask was shaken well, $1 \mathrm{~mL}$ solution containing $\mathrm{K}^{+}, \mathrm{Na}^{+}, \mathrm{Ca}^{2+}, \mathrm{Mg}^{2+}, \mathrm{Zn}^{2+}, \mathrm{Al}^{3+}, \mathrm{Fe}^{3+}, \mathrm{Cu}^{2+}, \mathrm{Pb}^{2+}, \mathrm{Mn}^{2+}$, $\mathrm{Ni}^{2+}, \mathrm{Co}^{2+}, \mathrm{Cd}^{2+}, \mathrm{Hg}^{2+}$ or $\mathrm{Cr}^{6+}$ ions of $30 \mu \mathrm{M}$ was added respectively. Then, the intensity of the fluorescence emission spectra $(F)$ was measured at room temperature and contrasted with the fluorescence intensity of the blank solution without metal ions $\left(F_{0}\right)$ to determine the selectivity of NCC-EDA for $\mathrm{Pb}^{2+}$. Afterwards, a series of $\mathrm{Pb}^{2+}$ ion solutions with different concentrations (0-50 $\mu \mathrm{M})$ were prepared, and a small amount of the solution was dropped into the dilution of phosphate buffer of NCC-EDA $(2 \mathrm{~mL}$ phosphate buffer of NCC-EDA diluted with distilled water to 4 $\mathrm{mL}$ ). After the resulting solution was mixed well, its intensity of fluorescence emission spectra was measured and the sensitivity of NCC-EDA for $\mathrm{Pb}^{2+}$ was determined by observing the change of the light intensity $(F)$. The sensitivity and selectivity measurements were conducted in triplicate.

\section{Result and discussion}

\subsection{Morphology, composition and microstructure of NCC- EDA}

Fig. 1 shows the TEM images of the aqueous dispersions of NCC and NCC-EDA.

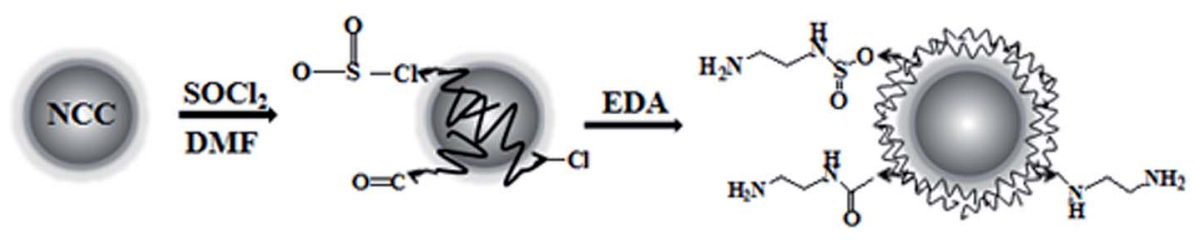

Scheme 1 Preparation process of the cellulose based fluorescent nanoparticles. 

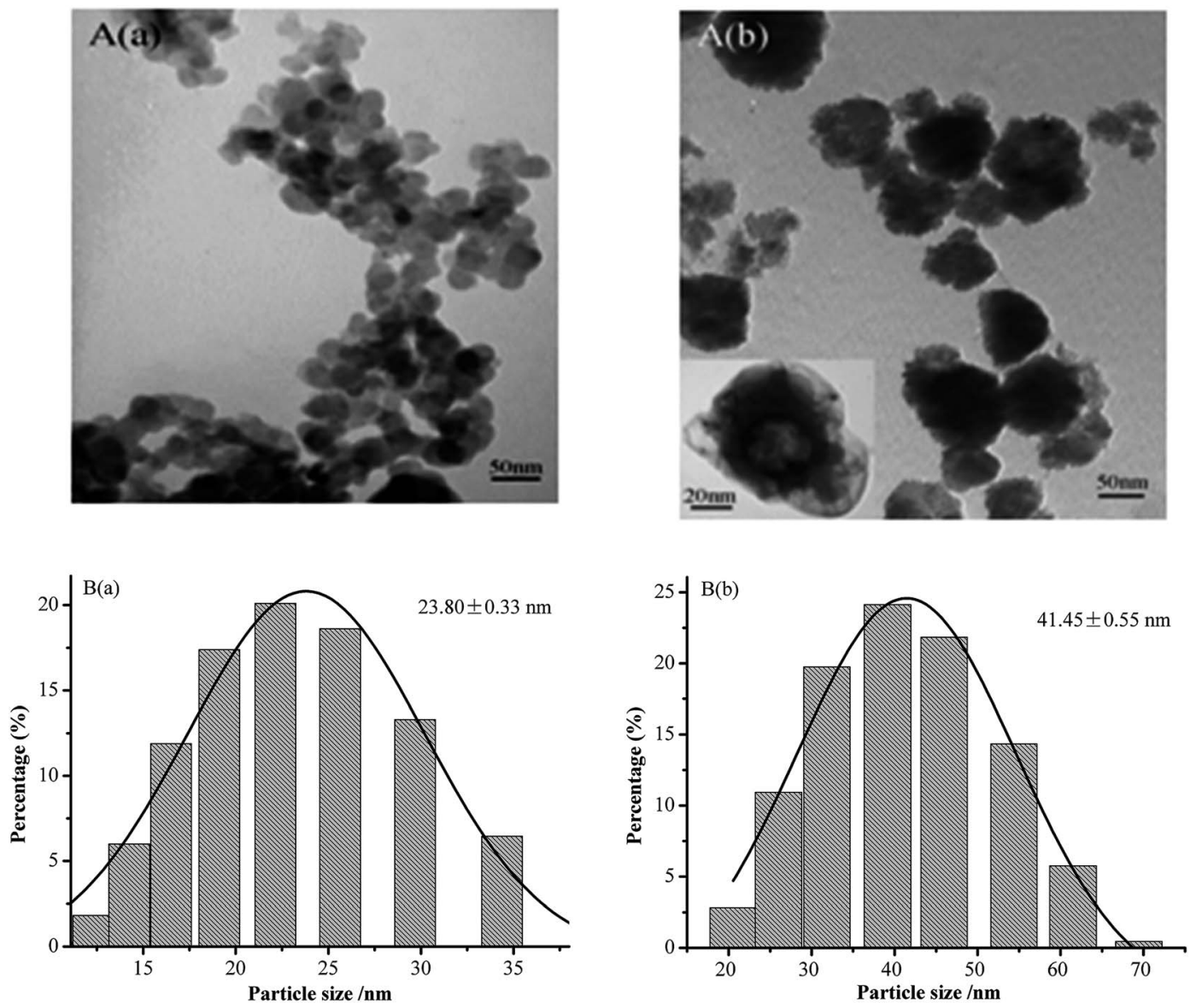

Fig. 1 TEM images (A) and size distribution (B) of (a) NCC and (b) NCC-EDA.

As can be seen, the NCC exists in the form of spherical particles with particle sizes of $23.80 \pm 0.33 \mathrm{~nm}$, and the formation process includes hydrolysis, dehydrated carbonization, and sulfonation of microcrystalline cellulose under the actions of mixed acids $\left(\mathrm{H}_{2} \mathrm{SO}_{4}+\mathrm{HCl}\right)$ and ultrasound. However, the NCC-EDA modified by EDA are vesicle-like spherical particles (which can be clearly seen from the TEM image magnified by 200000 times (see inset)). They are formed by the linking and intertwining effects of EDA on the $\mathrm{SOCl}_{2}$-treated NCC, and their particle size is $41.45 \pm 0.55 \mathrm{~nm}$, about twice the NCC. In addition, NCC-EDA particles can be well dispersed in water instead of aggregating together because of EDA modification.

Table 1 lists the compositions and testing results of the surface characteristics for NCC, NCC-Cl and NCC-EDA.
As the elemental analysis results (Table 1) show, NCC contains a small amount of S, probably due to the introduction of sulfate groups generated by the reaction between sulfuric acid and cellulose. After treatment with $\mathrm{SOCl}_{2}$, the contents of $\mathrm{C}, \mathrm{H}$ and $\mathrm{O}$ in $\mathrm{NCC}-\mathrm{Cl}$ all decrease, while those of $\mathrm{S}$ and $\mathrm{N}$ both increase and $\mathrm{Cl}$ appears. These indicate that the intermediates from the reaction of NCC with $\mathrm{SOCl}_{2}$ contain both R-Cl and R$\mathrm{SO}_{2} \mathrm{Cl}$, whereas the increase of the $\mathrm{N}$ content would probably be due to the remaining DMF in the system. After the intermediates react with EDA, the contents of $\mathrm{C}, \mathrm{H}$, and $\mathrm{N}$ in the product significantly increase, those $\mathrm{S}$ and $\mathrm{Cl}$ both decrease, while the $\mathrm{O}$ content remains almost the same, indicating that EDA has replaced most of the $-\mathrm{Cl}$ and $-\mathrm{SO}_{2} \mathrm{Cl}$ groups and is linked to NCC. Testing results of the surface characteristics of NCC and

Table 1 Compositions and testing results of the surface characteristics of NCC, NCC-Cl and NCC-EDA ${ }^{a}$

\begin{tabular}{|c|c|c|c|c|c|c|c|c|c|}
\hline Sample & $\mathrm{C}$ & $\mathrm{H}$ & $\mathrm{N}$ & $S$ & $\mathrm{O}$ & $\mathrm{Cl}$ & $S\left(\mathrm{~m}^{2} \mathrm{~g}^{-1}\right)$ & $R(\mathrm{~nm})$ & $V\left(\mathrm{~cm}^{3} \mathrm{~g}^{-1}\right)$ \\
\hline $\mathrm{NCC}-\mathrm{Cl}$ & 38.15 & 5.41 & 2.79 & 2.26 & 35.26 & 16.13 & - & - & - \\
\hline NCC-EDA & 42.33 & 7.12 & 13.12 & 0.92 & 36.11 & 0.4 & 25.318 & 36.815 & $1.136 \times 10^{-2}$ \\
\hline
\end{tabular}

${ }^{a}$ By difference, $\mathrm{C} \%+\mathrm{H} \%+\mathrm{N} \%+\mathrm{S} \%+\mathrm{O} \%+\mathrm{Cl} \%=100 \%$. 

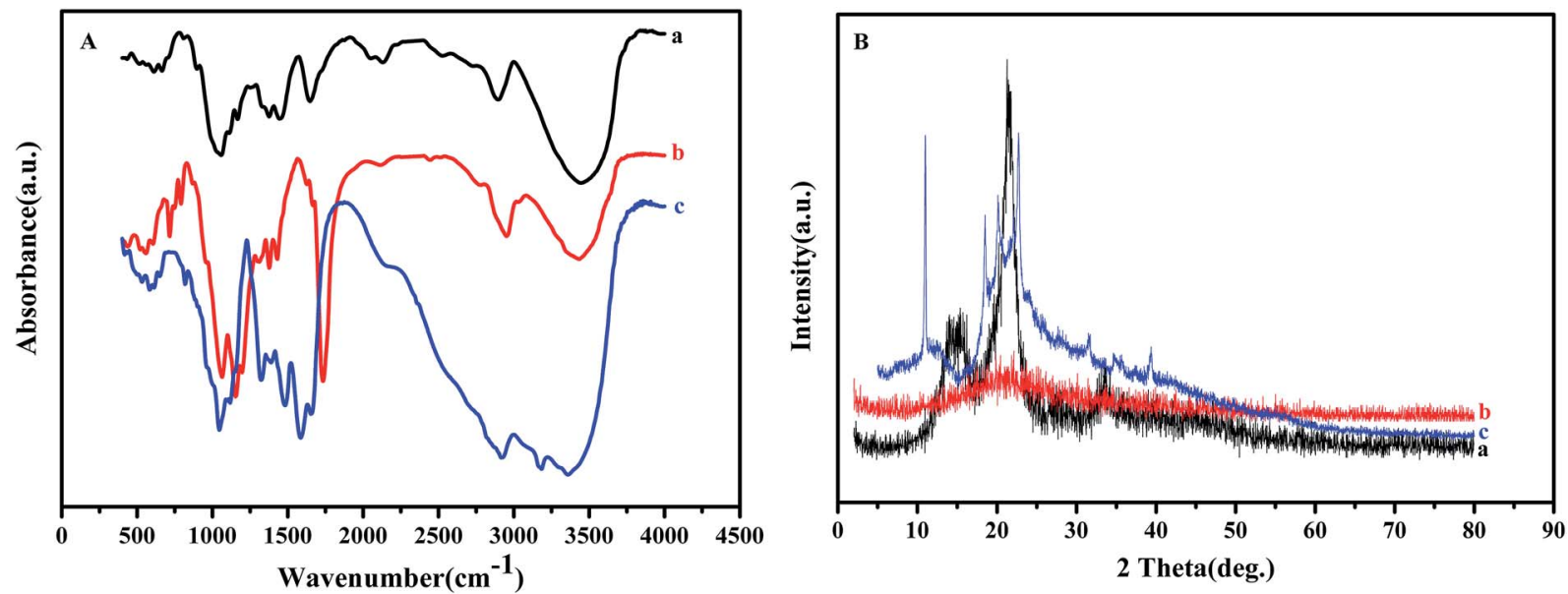

Fig. 2 FT-IR (A) and XRD (B) results of (a) NCC, (b) NCC-Cl and (c) NCC-EDA.

NCC-EDA show that the specific surface area, pore size and total pore volume of NCC-EDA are larger than those of NCC, demonstrating that NCC-EDA are particles with porous structure, which is consistent with the TEM results. This type of structure was probably formed by the cross-linking of EDA on the surface of NCC.

To determine the microstructure of the product, FT-IR, XRD and TG-DTA measurements were performed and the results are shown in Fig. 2 and 3.

Fig. 2(A) shows the FT-IR spectra of NCC, NCC-Cl and NCCEDA. The FT-IR spectrum of NCC (curve a) exhibits distinct spectral characteristics of cellulose. ${ }^{16}$ To be specific, the peak at $3445 \mathrm{~cm}^{-1}$ corresponds to the stretching vibration band of the hydroxyl group in cellulose; the peak at $2896 \mathrm{~cm}^{-1}$ is attributed to the stretching vibration band of methylene group $\left(-\mathrm{CH}_{2}-\right)$; a series of peaks between 1058 and $1167 \mathrm{~cm}^{-1}$ represent the bending vibration band of the ether bond $(\mathrm{C}-\mathrm{O}-\mathrm{C})$ in cellulose; the peak at $1647 \mathrm{~cm}^{-1}$ is indicative of the absorption band of the hydrogen bonding between the hydroxyl groups and free water; the band between 1444 and $1337 \mathrm{~cm}^{-1}$ reveals the existence of the crystalline structure of cellulose.

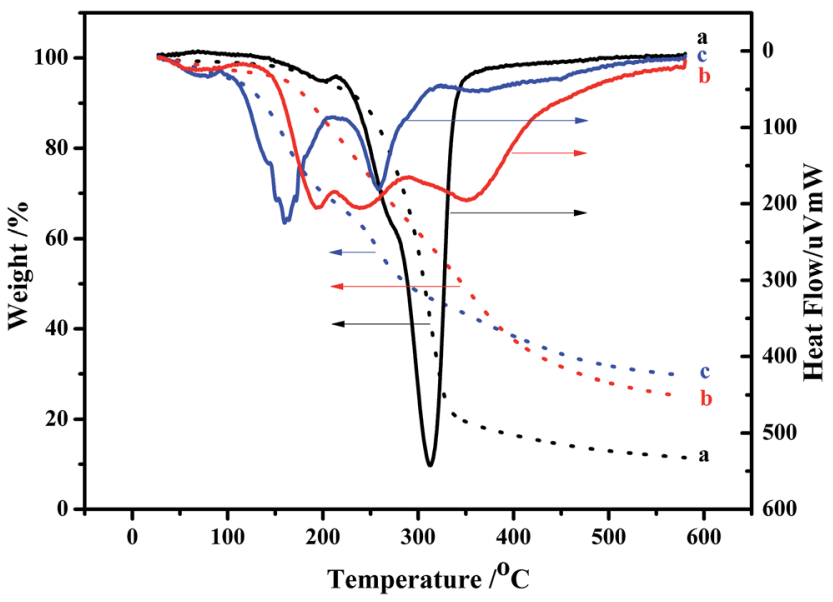

Fig. 3 TG-DTA results of (a) NCC, (b) NCC-Cl and (c) NCC-EDA.
Compared with that of NCC, in the FT-IR spectrum of the product obtained by $\mathrm{SOCl}_{2}$ chlorination (curve b), the intensity of the absorption peak of hydroxyl group at around $3400 \mathrm{~cm}^{-1}$ decreases, the peaks corresponding to the absorption bands of $\mathrm{S}=\mathrm{O}$ and $-\mathrm{SO}_{2}$ groups in sulfite appear at $1195 \mathrm{~cm}^{-1}$ and $1311 \mathrm{~cm}^{-1}$ respectively, and the characteristic peaks of the carbon-halogen bond C-Cl show up at $791 \mathrm{~cm}^{-1}, 753 \mathrm{~cm}^{-1}$, and $716 \mathrm{~cm}^{-1}$. These results are probably attributed to the generation of cellulose sulfonyl chloride $\mathrm{R}-\mathrm{SO}_{2} \mathrm{Cl}$ and $\mathrm{HCl}$ through the replacement of hydrogen in the hydroxyl group of NCC by SOCl in $\mathrm{SOCl}_{2}$, and the generation of chlorinated cellulose $\mathrm{R}-\mathrm{Cl}$ by partial removal of $\mathrm{SO}_{2}$ from $\mathrm{R}-\mathrm{SO}_{2} \mathrm{Cl}^{17}$ In addition, the peak corresponding to the stretching vibration absorption of $\mathrm{C}=\mathrm{O}$ and $\mathrm{C}=\mathrm{C}$ bonds appears at $1734 \mathrm{~cm}^{-1}$ in curve (b), probably due to the further oxidization and dehydrated carbonization of NCC caused by excess $\mathrm{SOCl}_{2}{ }^{18,19}$

Compared with curve (b), in the FT-IR spectrum of NCC-EDA (curve c), besides the distinct characteristic peaks of amide group (CONH-) at $3359 \mathrm{~cm}^{-1}$ and $3184 \mathrm{~cm}^{-1}$, the absorption peaks of band I, II and III of amide group appear at $1653 \mathrm{~cm}^{-1}$, $1585.2 \mathrm{~cm}^{-1}$ and $1323.4 \mathrm{~cm}^{-1}$ respectively; meanwhile, the stretching vibration peaks of $\mathrm{C}-\mathrm{Cl}$ at $791 \mathrm{~cm}^{-1}$ and $716 \mathrm{~cm}^{-1}$, and the stretching vibration peaks of $\mathrm{C}=\mathrm{O}$ and $\mathrm{C}=\mathrm{C}$ bonds at $1734 \mathrm{~cm}^{-1}$ and $1627 \mathrm{~cm}^{-1}$ all disappear, while the absorption peaks at $819 \mathrm{~cm}^{-1}$ and $650 \mathrm{~cm}^{-1}$ corresponding to the $\mathrm{O}-\mathrm{S}$ bond still exist, demonstrating that amination product has been successfully generated by the reaction of EDA with the intermediates.

Fig. 2(B) shows the XRD patterns of NCC, NCC-Cl and NCCEDA. NCC exhibits adjacent diffraction peaks at $2 \theta$ between $14.2^{\circ}$ and $15.6^{\circ}$ corresponding to (101) crystal plane; meanwhile, a sharp diffraction peak at $21.5^{\circ}$ corresponding to (002) crystal plane, and a diffraction peak at $33.4^{\circ}$ corresponding to (040) crystal plane also appear (curve a). These diffraction characteristics belong to a crystalline structure of typical cellulose I, which indicates that the cellulose in NCC is in highlyordered aggregation state via strong hydrogen bonding between hydroxyl groups. ${ }^{20}$ However, after treatment with $\mathrm{SOCl}_{2}$, the degree of crystallinity of the intermediates is 
fundamentally changed and the product turns into amorphous structure completely (curve b). This change can be attributed to the following two aspects: on the one hand, NCC is further degraded by the penetration and corrosion of acidic modification reagent; on the other hand, the intramolecular hydrogen bonding between NCC molecular chains is significantly weakened due to the introduction of the chloride ion and the intramolecular dehydration of cellulose.

Although the crystalline region of NCC is severely destroyed after chlorination, the degree of freedom of molecules will increase, which can enhance the possibility of the recombination of molecular chains. Therefore, after EDA treatment, the diffraction peaks for (101) and (040) planes completely disappear, and NCC-EDA exhibit diffraction peaks corresponding to the structure of cellulose III at $2 \theta=11.2^{\circ}, 18.4^{\circ}, 20.2^{\circ}$ and $22.7^{\circ}$. These demonstrate that EDA treatment leads to the recrystallization of the completely disrupted cellulose structure and promotes the disordered region to gradually form the structure of cellulose III (curve c). ${ }^{21}$ Moreover, the modification reagent may penetrate into the interior of the cellulose crystalline structure, which can change the cellulose crystal form and enlarge the interplanar spacing; meanwhile, EDA molecules bonded onto the cellulose surfaces may cross-link with each other and form new diffraction planes. As can be seen from curve (c), the degree of crystallinity of NCC-EDA decreases relative to that of NCC; the peak position of (101) plane shifts to smaller angle, and new diffraction peaks appear in the range of $2 \theta=31.5-39.4^{\circ}$.

Fig. 3 shows the TG-DTA measurement results of NCC, NCC$\mathrm{Cl}$ and NCC-EDA.

In general, MCC is observed to decompose in the temperature range of $250-420{ }^{\circ} \mathrm{C}$, and the maximum weight loss peak starts at $350{ }^{\circ} \mathrm{C}^{22}$ However, due to the smaller particle size of NCC relative to that of MCC, higher surface area can be exposed to heat. ${ }^{23}$ Additionally, the sulfate end groups from acid hydrolysis for NCC extraction have higher susceptibility to oxidative degradation. ${ }^{24}$ As a result, the NCC prepared herein decomposes at significantly lower temperatures of $150-350{ }^{\circ} \mathrm{C}$, the major loss starts at around $215^{\circ} \mathrm{C}$ and ends at about $350{ }^{\circ} \mathrm{C}$, and the maximum degradation temperature of NCC decreases to $312{ }^{\circ} \mathrm{C}$ (curve a).

Due to the increase of sulfate groups, the weight loss of NCC-Cl starts at about $130^{\circ} \mathrm{C}$ (curve b), which is earlier than that of NCC. Combined with the analysis results of XRD, it can be determined that the structure of the reaction intermediates between NCC and $\mathrm{SOCl}_{2}$ has already loosened, thus decreasing the degree of crystallinity. However, at $600{ }^{\circ} \mathrm{C}$, the weight loss ratio of $\mathrm{NCC}-\mathrm{Cl}$ is lower than that of NCC, and the content of remaining carbon is $25 \%$, indicating the occurrence of oxidation, dehydrated carbonization in the cellulose during $\mathrm{SOCl}_{2}$ treatment. ${ }^{25,26}$

The major weight loss of NCC-EDA takes place in two stages (curve c). In the first stage, the weight loss occurs between 107$204{ }^{\circ} \mathrm{C}$, which is caused by the pyrolysis of EDA groups linked to the cellulose surfaces; in the second stage, the weight loss occurs between $225-320{ }^{\circ} \mathrm{C}$, which corresponds to the weight loss peak of the main chain of cellulose. The major weight loss peaks of NCC-EDA start earlier than those of NCC, but the undecomposed residue accounts for $29.3 \%$ at $600{ }^{\circ} \mathrm{C}$. This can be attributed to the following two aspects: on the one hand, the main chain of NCC-EDA with the structure of cellulose III obtained through chlorination, oxidation, dehydrated carbonization and ethylenediamine processing has turned into cellulose II with stronger hydrogen bonding after heating, ${ }^{27}$ which requires more energy to accomplish its complete pyrolysis; on the other hand, the oxygen-containing groups doped with sulfur, chlorine and nitrogen, which are introduced into NCCEDA by the chlorination of thionyl chloride and the deactivation of ethylenediamine, may play a role of flame retardant. ${ }^{28}$

To further confirm the above results, the surface state of the NCC-EDA was analyzed by XPS and the results are shown in Fig. 4.

As can be seen from Fig. 4(A), five characteristic peaks of NCC-EDA appear at 531.0 eV, $399.8 \mathrm{eV}, 285.0 \mathrm{eV}, 231.7 \mathrm{eV}$ and $168.1 \mathrm{eV}$, which correspond to $\mathrm{O}_{1 \mathrm{~s}}, \mathrm{C}_{1 \mathrm{~s}}, \mathrm{~N}_{1 \mathrm{~s}}, \mathrm{~S}_{2 \mathrm{p}}$ and $\mathrm{Cl}_{2 \mathrm{p}}$, respectively. This indicates that NCC-EDA is mainly composed of five elements including carbon, nitrogen, oxygen, sulfur and chlorine, respectively with a content of $52.41 \%, 13.75 \%$, $29.08 \%, 4.11 \%$, and $0.65 \%$. The XPS results basically agree with the elemental analysis result above, demonstrating that the surface of NCC-EDA has a large number of functional groups containing oxygen, nitrogen and sulfur, together with a small amount of chlorine probably from incomplete substitution of $-\mathrm{SO}_{2} \mathrm{Cl}$ by EDA.

Fig. 4(B) shows the high-resolution XPS spectrum of $\mathrm{C}_{1 \mathrm{~s}} \cdot \mathrm{C}_{1 \mathrm{~s}}$ peak splits into three adjacent peaks at $283.6 \mathrm{eV}, 285.1 \mathrm{eV}$ and $286.5 \mathrm{eV}$, which correspond to $\mathrm{C}-\mathrm{C} / \mathrm{C}=\mathrm{C}, \mathrm{C}-\mathrm{S}$ and $\mathrm{C}-\mathrm{N}$ bonds, ${ }^{29,30}$ respectively. In the high-resolution XPS spectrum of $\mathrm{N}_{1 \mathrm{~s}}$ (Fig. 4(C)), $\mathrm{N}_{1 \mathrm{~s}}$ peak splits into two adjacent peaks at $398.2 \mathrm{eV}$ and $400.1 \mathrm{eV}$, which correspond to amide bond (CONH-) and aminolysis positive charge $-\mathrm{NH}^{3+}$, respectively. This result is consistent with the FT-IR result, demonstrating that EDA has been successfully linked to the NCC surface. In the highresolution XPS spectrum of $S_{2 p}$ (Fig. 4(D)), $S_{2 p}$ peak splits into two adjacent peaks at $166.9 \mathrm{eV}$ and $168.1 \mathrm{eV}$, which are characteristic peaks representing some sulfur-containing oxides (-C$\left.\mathrm{SO}_{x}-(x=2,3,4)\right) .{ }^{29}$ Combined with the analysis results above, it can be deduced that the two peaks are respectively from the sulfate group introduced by the reaction between $\mathrm{H}_{2} \mathrm{SO}_{4}$ and the hydroxyl groups in NCC, and the intermediate sulfonyl chloride groups generated by the reaction between $\mathrm{NCC}$ and $\mathrm{SOCl}_{2}$.

\subsection{Optical property of the NCC-EDA}

Fig. 5 shows the absorption and fluorescence emission spectra of the NCC-EDA aqueous dispersion. It can be seen that the NCC-EDA exhibits excellent absorption and fluorescence emission abilities.

As the UV-Vis absorption spectrum presents, NCC-EDA has absorption in both ultraviolet and visible light regions, and exhibits a distinct absorption band at about $340 \mathrm{~nm}$. When the aqueous dispersion of NCC-EDA is excited by the incident light of 350-500 $\mathrm{nm}$ in wavelength, fluorescence emission peaks appear between $448-550 \mathrm{~nm}$, which can be attributed to the surface modification of cellulose nanoparticles. ${ }^{31,32}$ From the 

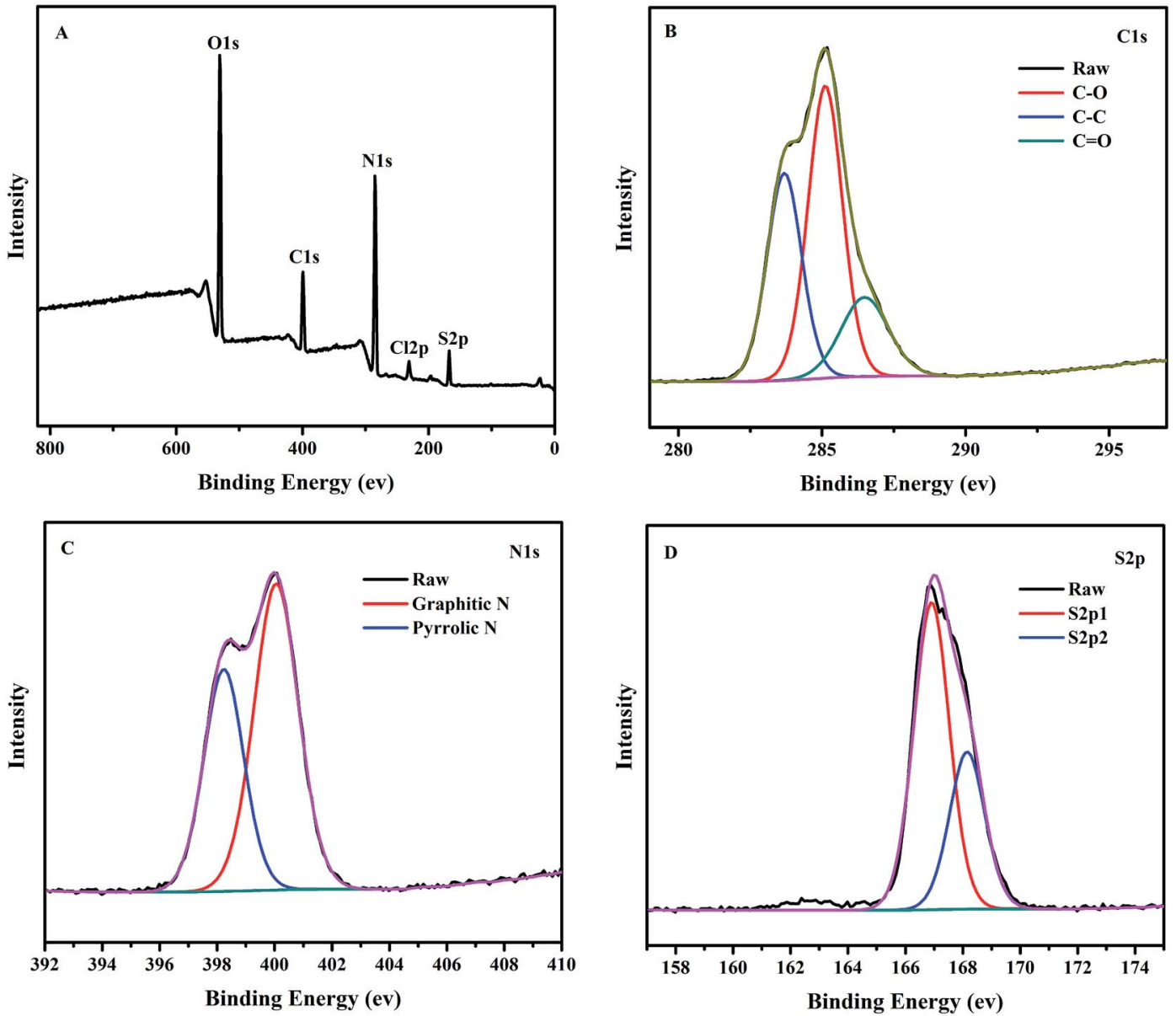

Fig. 4 X-ray photoelectron spectroscopy (XPS) of the NCC-EDA.

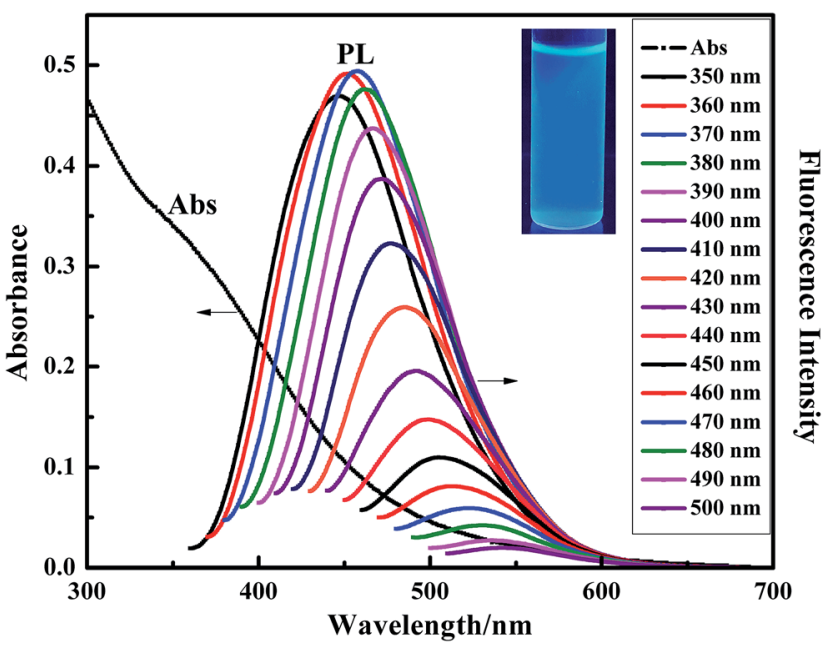

Fig. 5 Adsorption and fluorescence emission spectra of the NCC-EDA.

aforementioned experimental results, it can be known that, during the preparation of NCC-EDA, oxygen-containing groups doped with sulfur and nitrogen atoms have been successfully introduced into NCC-EDA due to the chlorination of thionyl chloride and the deactivation of ethylenediamine. These groups can not only generate chromophores on the surface of NCCEDA, but also act as energy trap sites to trap excitons and induce transitions, thus emitting fluorescence. In addition, with the increase of excitation wavelength, the fluorescence intensity increases first and then decreases; meanwhile gradual redshift can be observed. The optimal excitation wavelength is achieved at $360 \mathrm{~nm}$, and the strongest emission peak appears at about $452 \mathrm{~nm}$. The fluorescence spectral characteristics of NCC-EDA are basically the same as those of other typical luminescent carbon nanoparticles, which depend on the excitation wavelength and have spectral characteristics of multiple excitation and multiple emission. ${ }^{32-35}$ Moreover, the fluorescence quantum yield of the NCC-EDA aqueous dispersion is determined as $36.82 \%$ by using quinine sulfate as reference. Hence, it is easy to observe the strong and stable blue-green fluorescence emitted by the NCC-EDA aqueous dispersion under the UV lamp of $365 \mathrm{~nm}$ in wavelength (see inset of Fig. 5).

\subsection{Trace detection of $\mathrm{Pb}^{2+}$ ions by NCC-EDA as fluorescent probe}

Fluorescent nanoparticles are similar to semiconductor quantum dots, which can change the recombination efficiency of electronhole pair on the surface and result in the enhancement or 

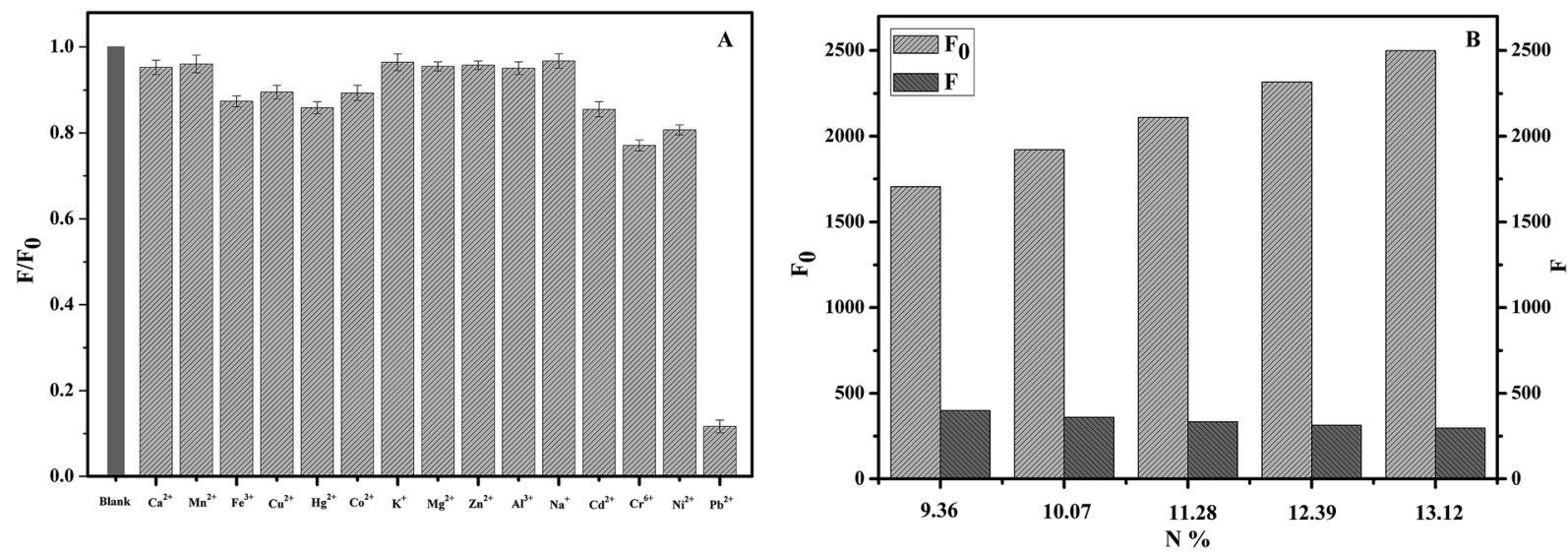

Fig. 6 (A) Selectivity of the NCC-EDA as a probe for metal ions in phosphate buffer; (B) effect of $\mathrm{Pb}^{2+}$ on the fluorescence quenching of NCCEDA with different amino content ( $y$-axis represents the ratio of the fluorescence intensity of NCC-EDA with the addition of $\mathrm{Pb}{ }^{2+}(F)$ to that of $\mathrm{NCC}-\mathrm{EDA}$ without the addition of $\mathrm{Pb}^{2+}\left(F_{0}\right) ; \mathrm{pH}=7$; content of the NCC-EDA, $2.5 \mu \mathrm{g} \mathrm{mL}^{-1}$; concentration of metal ions, $30 \mu \mathrm{M}$ ).

quenching of fluorescence through interaction with the targets, thus realizing the qualitative or quantitative analysis of the targets. $^{36}$ The structure and property of the fluorescence nanoparticles prepared by different methods are significantly different, so that their specific fluorescence responses to ions or other substances may be different.

To test the fluorescence responsivity of NCC-EDA synthesized in this paper to metal ions, the effects of 15 common metal ions $\left(\mathrm{K}^{+}, \mathrm{Na}^{+}, \mathrm{Ca}^{2+}, \mathrm{Mg}^{2+}, \mathrm{Zn}^{2+}, \mathrm{Al}^{3+}, \mathrm{Fe}^{3+}, \mathrm{Cu}^{2+}, \mathrm{Pb}^{2+}, \mathrm{Mn}^{2+}, \mathrm{Ni}^{2+}, \mathrm{Co}^{2+}\right.$, $\left.\mathrm{Cd}^{2+}, \mathrm{Hg}^{2+}, \mathrm{Cr}^{6+}\right)$ on the fluorescence intensity of NCC-EDA were studied, and the results are shown in Fig. 6(A).

As can be seen, the presence of $\mathrm{Ca}^{2+}, \mathrm{Mn}^{2+}, \mathrm{K}^{+}, \mathrm{Mg}^{2+}, \mathrm{Zn}^{2+}$, $\mathrm{Al}^{3+}$ and $\mathrm{Na}^{+}$does not cause the fluorescence intensity of the NCC-EDA aqueous dispersion to drop. In the presence of $\mathrm{Fe}^{3+}$, $\mathrm{Cu}^{2+}, \mathrm{Hg}^{2+}, \mathrm{Co}^{2+}, \mathrm{Cd}^{2+}, \mathrm{Cr}^{6+}$ and $\mathrm{Ni}^{2+}$, the fluorescence intensity slightly decays but not to a large extent. In the presence of $\mathrm{Pb}^{2+}$, the intensity decreases significantly. These indicate that $\mathrm{Pb}^{2+}$ ions have certain selective fluorescence quenching effect on the fluorescence of NCC-EDA.

For clear the mechanism of the cellulose-based fluorescent carbon nanoparticles to $\mathrm{Pb}^{2+}$, a batch of NCC-EDA with different amino content ( $\mathrm{N}$ content) was synthesized and used to examine the effect of $\mathrm{Pb}^{2+}$ on the fluorescence quenching of it (Fig. 6(B)). Within the scope of the study, the fluorescence intensity of the NCC-EDA was increased with the amino content, however, after the presence of $\mathrm{Pb}^{2+}$, the fluorescence intensity of the NCC-EDA drops with the increase of amino content in the product. The results shows that the effect of $\mathrm{Pb}^{2+}$ ions on the fluorescence quenching of NCC-EDA is significant, the fluorescence quenching associated with the surface characteristics of NCC-EDA and the existence of a large number of oxygen-containing functional groups doped with $\mathrm{N}$ and $\mathrm{S}$ on the surface. These functional groups can result in the fluorescence quenching of NCC-EDA via strong chelation with $\mathrm{Pb}^{2+}$. In addition, excessive EDTA, a strong chelating agent, is added into the test solutions to chelate with the $\mathrm{Pb}^{2+}$ ions in the solution completely. It is found that the fluorescence intensity of the solution almost recovers to the intensity in the absence of $\mathrm{Pb}^{2+}$ ions. Thus it can be further confirmed that the fluorescence quenching of the NCC-EDA aqueous dispersion is caused by the action between $\mathrm{Pb}^{2+}$ and the amino functional groups on the surface of NCC-EDA.

Fig. 7 demonstrates the influence of $\mathrm{pH}$ values of the test solution and reaction time of the NCC-EDA with $\mathrm{Pb}^{2+}$ ions on the fluorescence intensity change ratio $\left(F / F_{0}\right)$ of the NCC-EDA.

As can be seen from Fig. 7(A), in the range of 1-8, the adjustment of $\mathrm{pH}$ value of the NCC-EDA aqueous dispersion has significant effect on the chelation of the NCC-EDA for $\mathrm{Pb}^{2+}$ ions. At lower $\mathrm{pH}$ values $(\mathrm{pH}<5)$, There may be a protonation occurs between the active sites of NCC-EDA with $\mathrm{H}^{+}$, positive charges repel $\mathrm{Pb}^{2+}$, the binding ability of the active sites of NCCEDA toward $\mathrm{Pb}^{2+}$ ions will decline, the fluorescence quenching of the NCC-EDA aqueous dispersion is limited; by contrast, when the $\mathrm{pH}$ value is higher than $7, \mathrm{~Pb}^{2+}$ may form hydroxide precipitates, the $\mathrm{Pb}^{2+}$ that can form chelate with NCC-EDA and make its fluorescence quenching will be reduced; when $\mathrm{pH}=7$, the fluorescence intensity of the NCC-EDA exhibits a larger decrease. This shows that the NCC-EDA and $\mathrm{Pb}^{2+}$ ions can form stable chelate under neutral condition. To achieve optimum chelation and prevent the hydroxide precipitation of $\mathrm{Pb}^{2+}$ ions, $\mathrm{pH}=7$ was selected as the optimum value for chelation ${\mathrm{of} \mathrm{Pb}^{2+}}^{2+}$ ions. Furthermore, the reaction between NCC-EDA and $\mathrm{Pb}^{2+}$ ions is fast, the fluorescence intensity of the NCC-EDA aqueous dispersion has a significant reduction within 3 min (Fig. 7(B)). This suggests that the nanoprobes with quick response prepared in this work hold great promise for real-time tracking of $\mathrm{Pb}^{2+}$ in environmental system.

Fig. 8(A) displays the dependence of fluorescence intensity change ratio $\left(F / F_{0}\right)$ of the NCC-EDA on the concentration of $\mathrm{Pb}^{2+}$ ions as well as the corresponding fluorescence spectra (see inset) after adding different concentrations of $\mathrm{Pb}^{2+}$ ions into the NCC-EDA aqueous dispersion. It can be seen that with the increase of $\mathrm{Pb}^{2+}$ concentration, $F / F_{0}$ gradually decreases. When $\mathrm{Pb}^{2+}$ concentration is above $30 \mu \mathrm{M}$, the value of $F / F_{0}$ tends to level off with further increase of $\mathrm{Pb}^{2+}$ concentration, which may be due to that the absorption of NCC-EDA toward $\mathrm{Pb}^{2+}$ ions has reached the saturated state. 

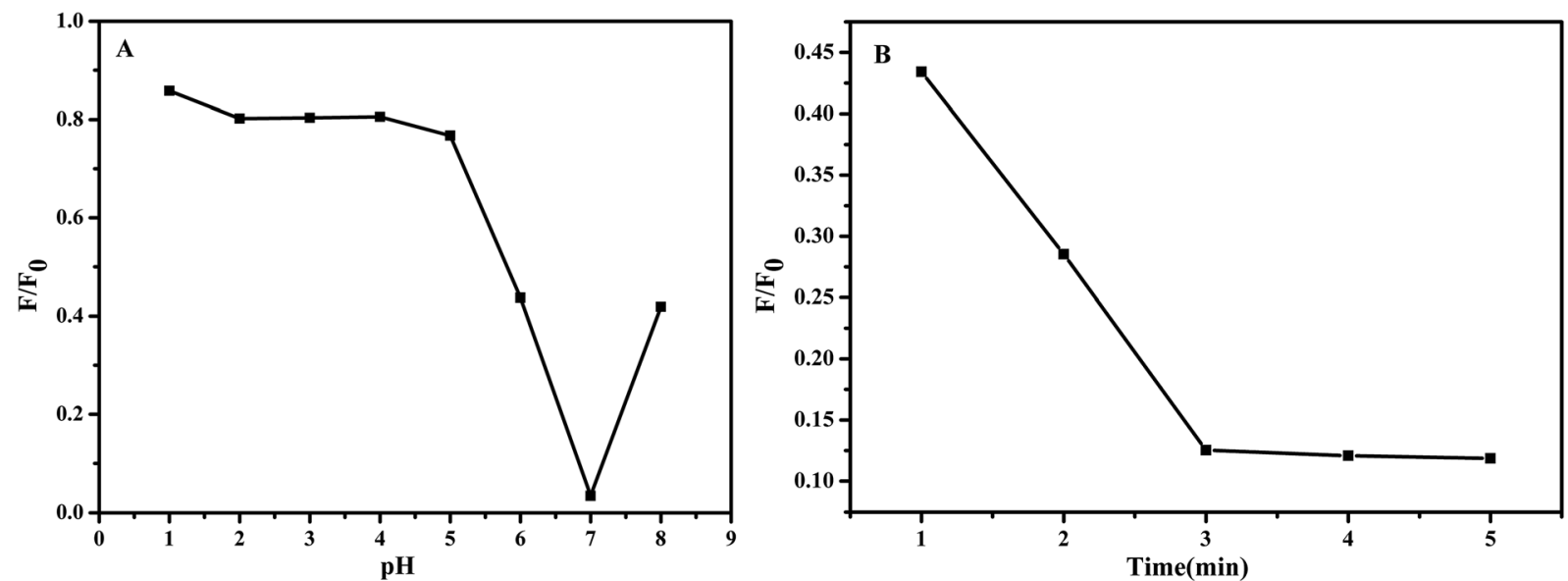

Fig. 7 Influence of $(A) \mathrm{pH}$ values of the test solution and (B) reaction time of the NCC-EDA with $\mathrm{Pb}^{2+}$ ions on the fluorescence intensity change ratio $\left(F / F_{0}\right)$ of the NCC-EDA aqueous dispersion.
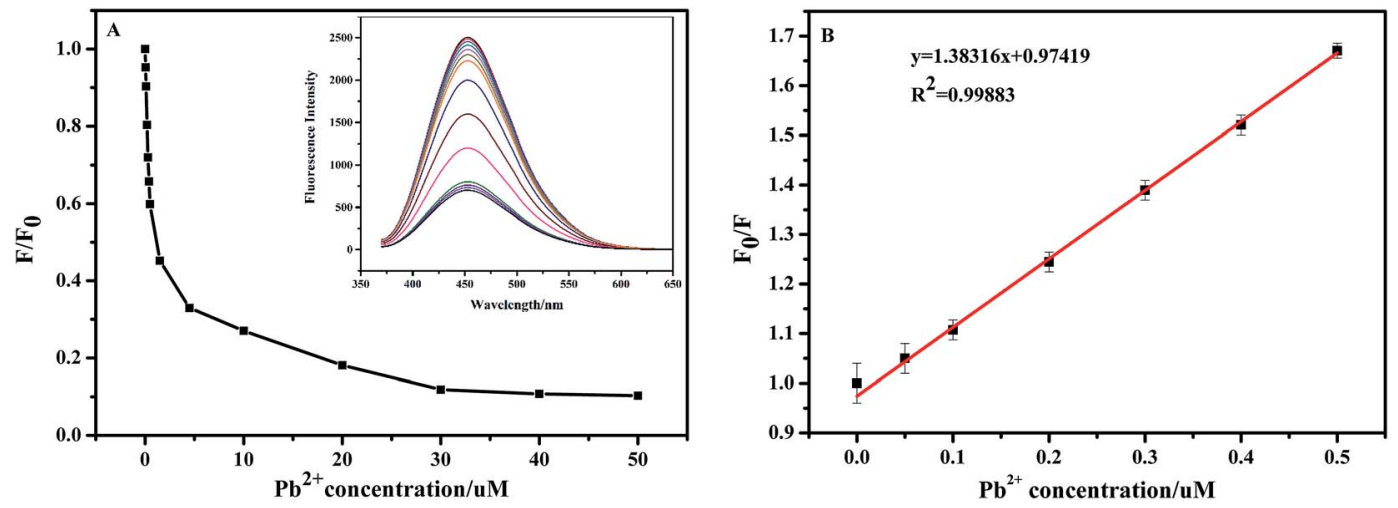

Fig. 8 (A) The dependence of $F / F_{0}$ on the concentration of $\mathrm{Pb}^{2+}$ in the range of $0-50 \mu \mathrm{M}$ (inset: the corresponding fluorescence response of the NCC-EDA dispersion upon the addition of various concentrations of $\mathrm{Pb}^{2+}$ ); (B) Stem-Volmer plot for the fluorescence quenching efficiency of the NCC-EDA at lower $\mathrm{Pb}^{2+}$ concentrations of $0.0187-0.5 \mu \mathrm{M}$.

Table 2 Figures of merit of recently reported methods for determination of $\mathrm{Pb}(॥)$

\begin{tabular}{llll}
\hline Methods/materials & Linear range $(\mu \mathrm{M})$ & Detection limit & Ref. \\
\hline GSH/CALNN-AuNPs & $1-10 \mu \mathrm{M}$ & $1 \mu \mathrm{M}$ & 37 \\
2-Mercaptoethanol-Au NPs- $\mathrm{Na}_{2} \mathrm{~S}$ & $0.05-0.5 \mu \mathrm{M}$ & $45 \mathrm{nM}$ & 38 \\
C-Dots & Not given & $5.05 \mu \mathrm{M}$ & 39 \\
$\mathrm{MnO}_{2} /$ carbon composites & Not given & $27 \mathrm{nM}$ & 40 \\
NCC-EDA & $0.0187-0.5 \mu \mathrm{M}$ & $24 \mathrm{nM}$ & This work
\end{tabular}

The fluorescence quenching efficiency of NCC-EDA at lower $\mathrm{Pb}^{2+}$ concentrations $(0.0187-0.5 \mu \mathrm{M})$ fits the Stern-Volmer equation: $F_{0} / F=1+K_{\mathrm{sv}}[\mathrm{Q}]$, in which $K_{\mathrm{sv}}$ is the Stern-Volmer quenching constant and $[\mathrm{Q}]$ represents the $\mathrm{Pb}^{2+}$ concentration. Within this low concentration range, $F_{0} / F$ is linear with $\mathrm{Pb}^{2+}$ concentration (see Fig. 8(B)), and the regression equation is $F_{0} / F=1.38316 x+0.97419\left(R^{2}=0.99883\right)$, with the lowest detection limit of $24 \mathrm{nM}$.

A comparison was made between our present work and those reported in literature for determination of $\mathrm{Pb}(\mathrm{II})$, which was shown in Table 2. As can be seen, the present work has high sensitivity, good linear response range and comparable detection limit to that found in other studies. This result demonstrates that the NCCEDA synthesized in this experiment can be used as fluorescent probe to detect the lead ions in aqueous solutions.

\section{Conclusion}

Cellulose-based fluorescent nanoparticles (NCC-EDA) with relatively high fluorescence quantum yield were prepared in 
this paper. The prepared NCC-EDA were vesicle-like porous particles formed by the linking and intertwining effects of EDA on the $\mathrm{SOCl}_{2}$-treated NCC. During preparation of the NCCEDA particles, oxygen-, sulfur- and nitrogen-functional groups are introduced via the chlorination with thionyl chloride and subsequent deactivation by using ethylenediamine. This generates chromophores on the surface of the NCC-EDA and sites to trap excitons and to induce transitions. Hence, aqueous dispersion of NCC-EDA that possess strong absorbance and fluorescence.

In the presence of trace $\mathrm{Pb}^{2+}$ ions, the prepared NCC-EDA showed selective fluorescence quenching, and the detection limit of $\mathrm{Pb}^{2+}$ ions in water was as low as $24 \mathrm{nM}$. It was believed that the NCC-EDA hold great promise for real-world sensor applications.

\section{Acknowledgements}

The authors are grateful for the financial support of the National Science Foundation of China (21663028).

\section{References}

1 S. C. Ray, A. Saha, N. R. Jana and R. Sarkar, J. Phys. Chem. C, 2009, 113, 18546-18551.

2 J. Schill, A. P. H. J. Schenning and L. Brunsveld, Macromol. Rapid Commun., 2015, 36, 76-83.

3 X. C. Sun, J. Mater. Chem. A, 2016, 4, 103893.

4 P. Zuo, D. Xiao, M. Gao, J. Peng, R. Pan, Y. Xia and H. He, Microchim. Acta, 2014, 181, 1309-1316.

5 W. Lu, X. Qin, S. Liu, G. Chang, Y. Zhang, Y. Luo, A. M. Asiri, A. O. Al-Youbi and X. Sun, Anal. Chem., 2012, 84, 5351-5357.

6 P. C. Hsu, Z. Y. Shih, C. H. Lee and H. T. Chang, Green Chem., 2012, 14, 917-920.

7 J. Zhou, Z. Sheng, H. Han, M. Zou and C. Li, Mater. Lett., 2012, 66, 222-224.

8 M. J. Krysmann, A. Kelarakis and E. P. Giannelis, Green Chem., 2012, 14, 3141-3145.

9 M. Saxena and S. Sarkar, Diamond Relat. Mater., 2012, 24, 11-14.

10 D. R. D. S. Souza, J. P. D. Mesquita, R. M. Lago, L. D. Caminhas and F. V. Pereira, Ind. Crops Prod., 2016, 1, 121-128.

11 H. Peng and J. Travassejdic, Chem. Mater., 2009, 21, 55635565.

12 Y. Yang, J. Cui, M. Zheng, C. Hu, S. Tan, Y. Xiao, Q. Yang and Y. Liu, Chem. Commun., 2011, 48, 380-382.

13 H. Li, X. He, Y. Liu, H. Huang, S. Lian, S. T. Lee and Z. Kang, Carbon, 2011, 49, 605-609.

14 J. Zhang, New J. Chem., 2010, 11, 1336-1339.
15 A. B. Bourlinos, A. Stassinopoulos, D. Anglos, R. Zboril, V. Georgakilas and E. P. Giannelis, Chem. Mater., 2008, 20, 4539-4541.

16 A. Alemdar and M. Sain, Bioresour. Technol., 2008, 99, 16641671.

17 K. Oka, Synthesis, 1981, 9, 661-681.

18 L. K. Ibrahim, S. M. Hussin and Y. M. Al-obaidi, Int. J. Mater. Chem. Phys., 2015, 1, 99-109.

19 M. Z. Fan, D. Dai and B. Huang, Fourier Transform-Materials Analysis, 2012, vol. 3, pp. 45-68.

20 D. Klemm, B. Heublein, H. P. Fink and A. Bohn, Angew. Chem., Int. Ed., 2005, 36, 3358-3393.

21 M. Lewin and L. G. Roldan, J. Polym. Sci., Part C: Polym. Symp., 1971, 36, 213-229.

22 S. Spoljaric, A. Genovese and R. A. Shanks, Composites, Part A, 2009, 40, 791-799.

23 R. S. Cheng and J. S. China, J. South China Univ. Technol., Nat. Sci., 2007, 35, 91-98.

24 K. A. Iyer, A. M. Flores and J. M. Torkelson, Polymer, 2015, 75, 78-87.

25 M. Roman and W. T. Winter, Biomacromolecules, 2004, 5, 1671-1677.

26 D. Y. Kim, Y. Nishiyama, M. Wada and S. Kuga, Cellulose, 2001, 8, 29-33.

27 A. Sarko and R. Muggli, Macromolecules, 1974, 7, 486-494.

28 S. Gaan and G. Sun, J. Anal. Appl. Pyrolysis, 2007, 78, 371-377.

29 C. Wang, RSC Adv., 2014, 4, 54060-54065.

30 X. Jin, X. Sun, G. Chen, L. Ding, Y. Li, Z. Liu, Z. Wang, W. Pan, C. Hu and J. Wang, Carbon, 2015, 81, 388-395.

31 R. Liu, D. Wu, S. Liu, K. Koynov, W. Knoll and Q. Li, Angew. Chem., Int. Ed., 2009, 48, 4598-4601.

32 Y. P. Sun, B. Zhou, Y. Lin, W. Wang, K. A. Shiral Fernando, P. Pathak, M. J. Mezinai, B. A. Harruff, X. Wang, H. Wang, P. G. Luo, H. Yang, M. E. Kose, B. Chen, L. M. Veca and M. Y. Xie, J. Am. Chem. Soc., 2006, 128, 7756-7757.

33 Y. Dong, H. Pang, H. B. Yang, C. Guo, J. Shao, Y. Chi, C. M. Li and T. Yu, Angew. Chem., Int. Ed., 2013, 52, 7800-7804.

34 C. Zhu, J. Zhai and S. Dong, Chem. Commun., 2012, 48, 93679369.

35 D. Sun, R. Ban, P. H. Zhang, G. H. Wu, J. R. Zhang and J. J. Zhu, Carbon, 2013, 64, 424-434.

36 F. Yan, Y. Zou, M. Wang, X. Mu, N. Yang and L. Chen, Sens. Actuators, B, 2014, 192, 488-495.

37 D. Zhu, X. Li, X. Liu, J. Wang and Z. Wang, Biosens. Bioelectron., 2012, 31, 505-509.

38 Y. L. Hung, T. M. Hsiung, Y. Y. Chen, Y. F. Huang and C. C. Huang, J. Phys. Chem. C, 2010, 114, 16329-16334.

39 S. S. Wee, Y. H. Ng and S. M. Ng, Talanta, 2013, 116, 71-76. 40 M. Mališić, A. Janošević, B. Š. Paunković, I. Stojković and G. Ćirić-Marjanović, Electrochim. Acta, 2012, 74, 158-164. 